\title{
ESTIMATION OF CHROMOSOMAL HOMOLOGY BETWEEN AEGILOPS SPELTOIDES AND THE TETRAPLOID WHEATS BY USING B-CHROMOSOMES ${ }^{1)}$
}

\author{
JUN'ICHI SANO AND MASATAKE TANAKA \\ Plant Germ-plasm Institute, Faculty of Agriculture \\ Kyoto University, Muko, Kyoto 617
}

Received July 13, 1979

\begin{abstract}
In order to estimate chromosomal homology between Aegilops speltoides (genomic constitution SS) and the tetraploid wheats (AABB or AAGG), effects of the B-chromosomes and genotypes of Ae. speltoides on chromosome pairing at $\mathrm{MI}$ of meiosis in $\mathrm{F}_{1}$ hybrids of Ae. speltoides $\times$ tetraploid wheats were investigated.

In hybrids with B's, chromosome pairing was strikingly affected by the B's and very small amount of pairing was observed. In hybrids without B's, however, extensive pairing was observed, although the amount of pairing varied due to the speltoides genotypes involved. In these hybrids with or without B's, although some minor yet distinctive differences in pairing attributable to the kind of genomic constitution of the wheat parent were observed in the multivalent frequency and in the distribution (or terminalization) of chiasmata, both hybrids with different genomic constitution (SAB or SAG) had essentially the same amount of pairing. Since the B-chromosomes used did not cause asynapsis (or desynapsis) of homologous chromosomes in Ae. speltoides, the pairing reduced by the presence of B's in the hybrids does not seem to be homologous and is probably homoeologous. Therefore, it can be concluded that there is little chromosomal homology between Ae. speltoides and both tetraploid wheats of genomic constitution $\mathrm{AABB}$ and AAGG.
\end{abstract}

\section{INTRODUCTION}

There are two kinds of genomic constitutions in the tetraploid wheats, i.e., AABB and AAGG (Lilienfeld and Kihara 1934). The A genome derives from one of the diploid wheats. But the origin of the second genome, B or G, is not known. Many workers suggested that the B genome donor was Aegilops speltoides Tausch (2x, genomic constitution SS), for which, Sarkar and Stebbins (1956) provided morphological evidence, Riley et al. (1958) showed karyotypic and geographical evidence, and Rees

1) Contribution No. 14 from the Plant Germ-plasm Institute, Faculty of Agriculture, Kyoto University, Japan. 
(1963) gave evidence from the amount of nuclear DNA. It is well known that there is partial homology between the B and G genomes. Examining the wild tetraploid wheats cytogenetically, Tanaka and Ishii (1973) suggested that both genomes had originated in the Zagros Mountains from chromosomal reorganization in the Ae. speltoides-Triticum boeoticum amphiploid.

Estimation of the chromosomal homology between Ae. speltoides and the tetraploid wheats is difficult, because the former suppresses the activity of the gene $P h$ on wheat chromosome 5BL and thus allows not only homologous but also homoeologous chromosome pairing to occur in hybrids (Riley et al. 1961). There may be two ways to overcome this difficulty. One is to find genotypes in Ae. speltoides which do not induce homoeologous pairing but permit homologous pairing. Genotypic variation to affect the amount of homoeologous pairing in hybrids with polyploid wheats has been reported in this species (Dvořák 1972; Kimber and Athwal 1972; Shands and Kimber 1973). Several strains were found to cause little or no homoeologous pairing in hybrids. One of these strains examined showed normal homologous pairing at both the diploid and colchicine-induced autotetraploid levels (Larsen and Kimber 1973). The other is to use B-chromosomes of Ae. speltoides (Simchen et al. 1971). Mcchizuki (1964) found that B-chromosomes of Ae. mutica did not affect homologous pairing but reduced homoeologous pairing in hybrids with wheat. Similar effects of B's were reported in interspecific hybrids of Lolium (Evans and Macefield 1972) and in Lolium perenne $\times$ Festuca arundinacea hybrids (Bowman and Thomas 1973). Therefore, the B's of Ae. speltoides may have a similar activity.

We attempted these two ways mentioned above and report the results here.

\section{MATERIALS AND METHODS}

Triticum dicoccoides Körn. $(2 \mathrm{n}=4 \mathrm{x}=28 ; \mathrm{KU}$ strain no. 110), T. dicoccum Schübl. $(2 \mathrm{n}=4 \mathrm{x}=28 ; 114,115)$ and $T$. durum Desf. $(2 \mathrm{n}=4 \mathrm{x}=28 ; 125)$ were used as the tetraploid wheats with AABB. T. araraticum Jakubz. $(2 \mathrm{n}=4 \mathrm{x}=28 ; 196-1,196-2,8601,8831)$ was used as the tetraploid wheat with AAGG. Twelve strains of Ae. speltoides without B-chromosomes and two strains with B's were also used. All these speltoides strains were originally collected by the Botanical Expedition to the Northern Highland of Mesopotamia, Kyoto University (1970), except one (5715) which was originally collected by the Botanical Mission of the University of Kyoto to the Eastern Mediterranean Countries (1959). All the materials used have been maintained by selfing at the Plant Germ-plasm Institute, Faculty of Agriculture, Kyoto University.

Each strain of $A e$. speltoides was crossed with at least one strain of the AABBwheat and one strain of AAGG-wheat. The hybrids were grown in glasshouse or under field conditions. But some hybrids were placed in a growth cabinet at $22^{\circ} \mathrm{C}$ with continuous light prior to meiosis. Ae. speltoides was grown under field conditions. Cytological observations were made at the first metaphase of meiosis in PMC's fixed in acetic alcohol $(1: 3)$ and processed by the aceto-carmine squash technique. 


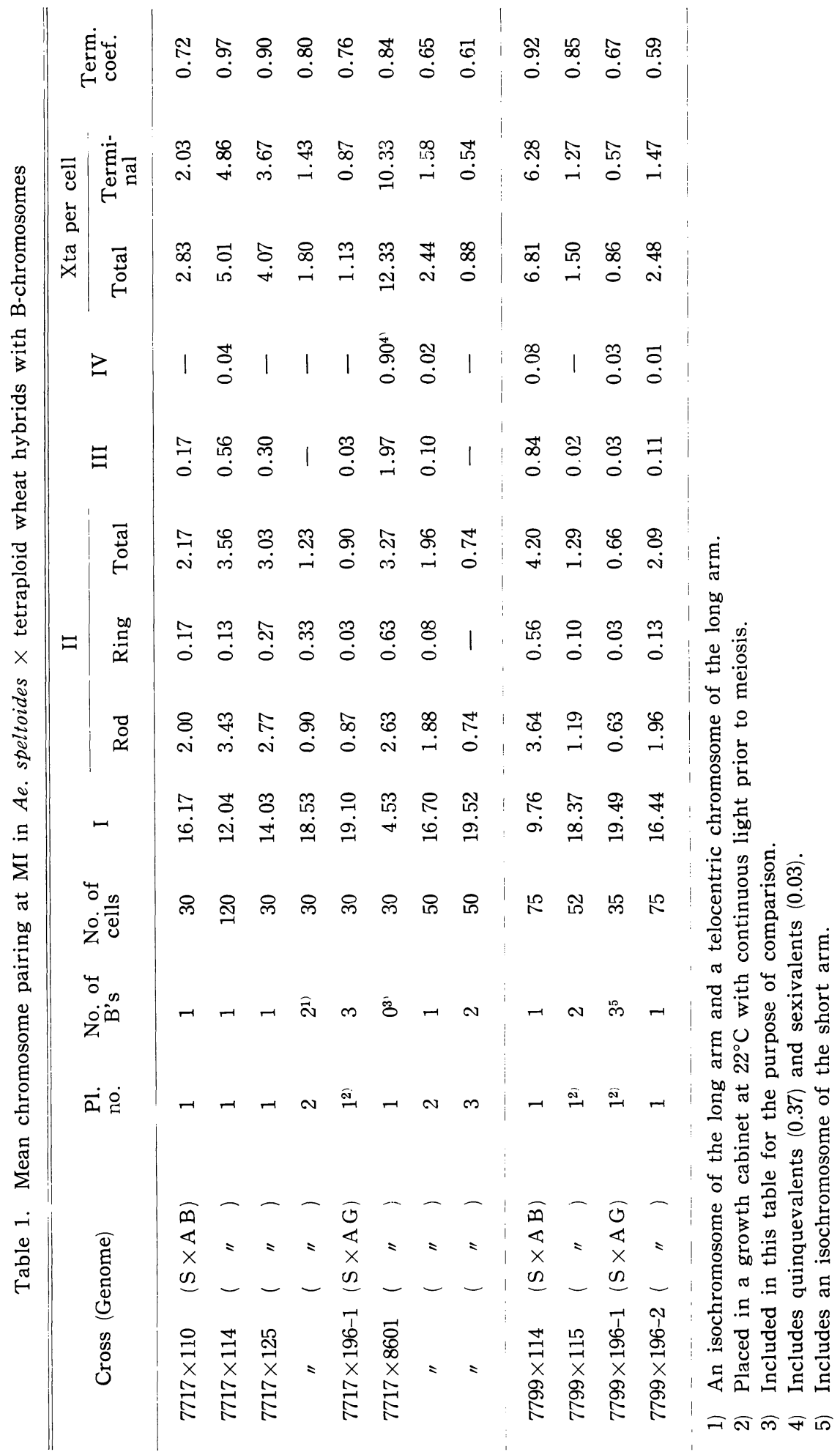


Table 2. Mean chromosome pairing at MI in Ae. speltoides $\times$ tetraploid wheat hybrids without B-chromosomes

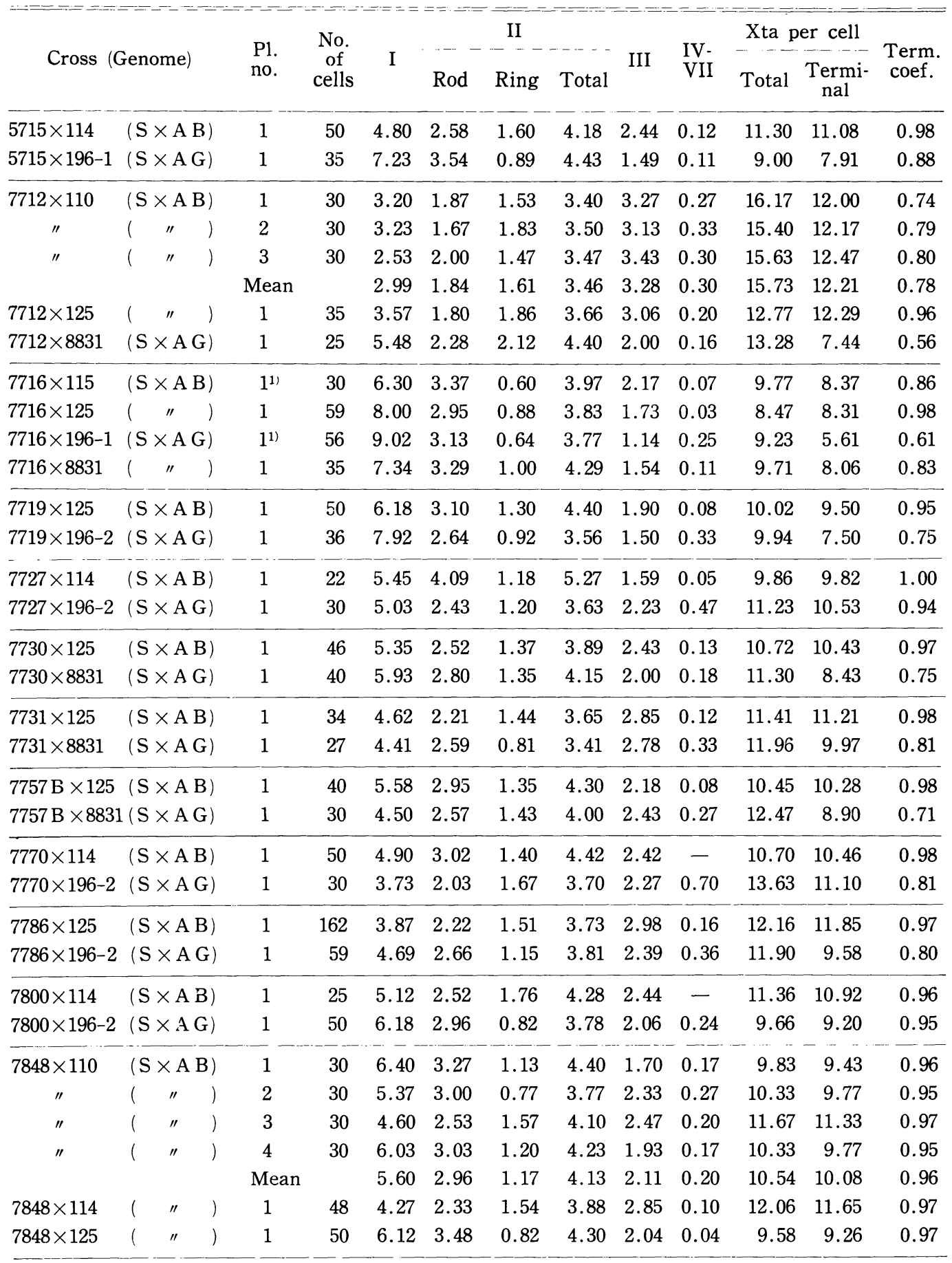


Table 2. (Continued)

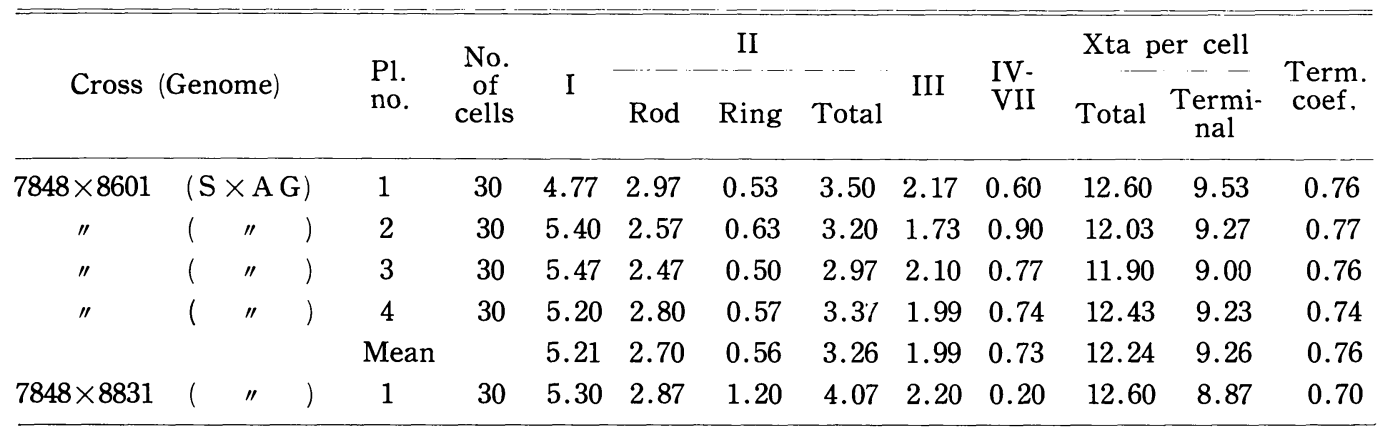

1) Placed in a growth cabinet at $22^{\circ} \mathrm{C}$ with continuous light prior to meiosis.

Table 3. Mean chromosome pairing at MI in Ae. speltoides with B-chromosomes

\begin{tabular}{|c|c|c|c|c|c|c|c|c|c|}
\hline \multirow{2}{*}{ Strain no. ${ }^{1)}$} & \multirow{2}{*}{$\begin{array}{l}\text { No., of } \\
\text { B's }\end{array}$} & \multirow{2}{*}{$\begin{array}{l}\text { No. of } \\
\text { cells }\end{array}$} & \multirow{2}{*}{ I } & \multicolumn{3}{|c|}{ II } & \multicolumn{2}{|c|}{ Xta per cell } & \multirow{2}{*}{$\begin{array}{l}\text { Term. } \\
\text { coef. }\end{array}$} \\
\hline & & & & Rod & Ring & Total & Total & Terminal & \\
\hline 7717 & 5 & 30 & - & 0.63 & 6.37 & 7.00 & 15.57 & 10.63 & 0.68 \\
\hline 7799 & 1 & 30 & 0.07 & 0.73 & 6.23 & 6.97 & 13.30 & 12.37 & 0.93 \\
\hline
\end{tabular}

1) Data were obtained from one plant.

\section{RESULTS AND DISCUSSION}

Mean chromosome pairing and chiasma frequency in the hybrids of Ae. speltoides $\times$ tetraploid wheats are given in Tables 1 and 2 .

The hybrids with various numbers of B-chromosomes showed extensive univalent formation (Figs. 1-4) and had high mean univalent frequencies per cell, the range of which was $9.76(7799 \times 114$, plant 1) to $18.53(7717 \times 125$, plant 2$)$ in the hybrids with genomic constitution $\mathrm{SAB}$ and $16.44(7799 \times 196-2$, plant 1$)$ to $19.52(7717 \times 8601$, plant 3$)$ in the SAG-hybrids (Table 1). Therefore, when the mean frequency was the highest, only $2.47(7717 \times 125$, plant 2$)$ and $1.48(7717 \times 8601$, plant 3$)$ chromosomes per cell showed pairing on the average in SAB- and SAG-hybrid, respectively. Since the B-chromosomes did not cause asynapsis (or desynapsis) of homologous chromosomes in both speltoides strains used (Table 3; Figs. 9, 10), they should not cause asynapsis (or desynapsis) of homologues in hybrids either. If this is true, these data indicate that the average number of chromosomes showing homologous pairing is at most 2.47 per cell in SABand 1.48 per cell in SAG-hybrid. This means that there is little chromosomal homology between $A e$. speltoides and the tetraploid wheats with AABB or AAGG.

In the hybrids without B's involving 13 speltoides strains, there was a considerable amount of pairing (Figs. 5-8). The range of mean univalent frequencies of these hybrids was $2.53(7712 \times 110$, plant 3$)$ to $9.02(7716 \times 196-1$, plant 1$)$ per cell (Table 2 and one hybrid in Table 1), which did not overlap with that of the hybrids with B's and significantly differed from it. In these 13 speltoides strains including one strain with 


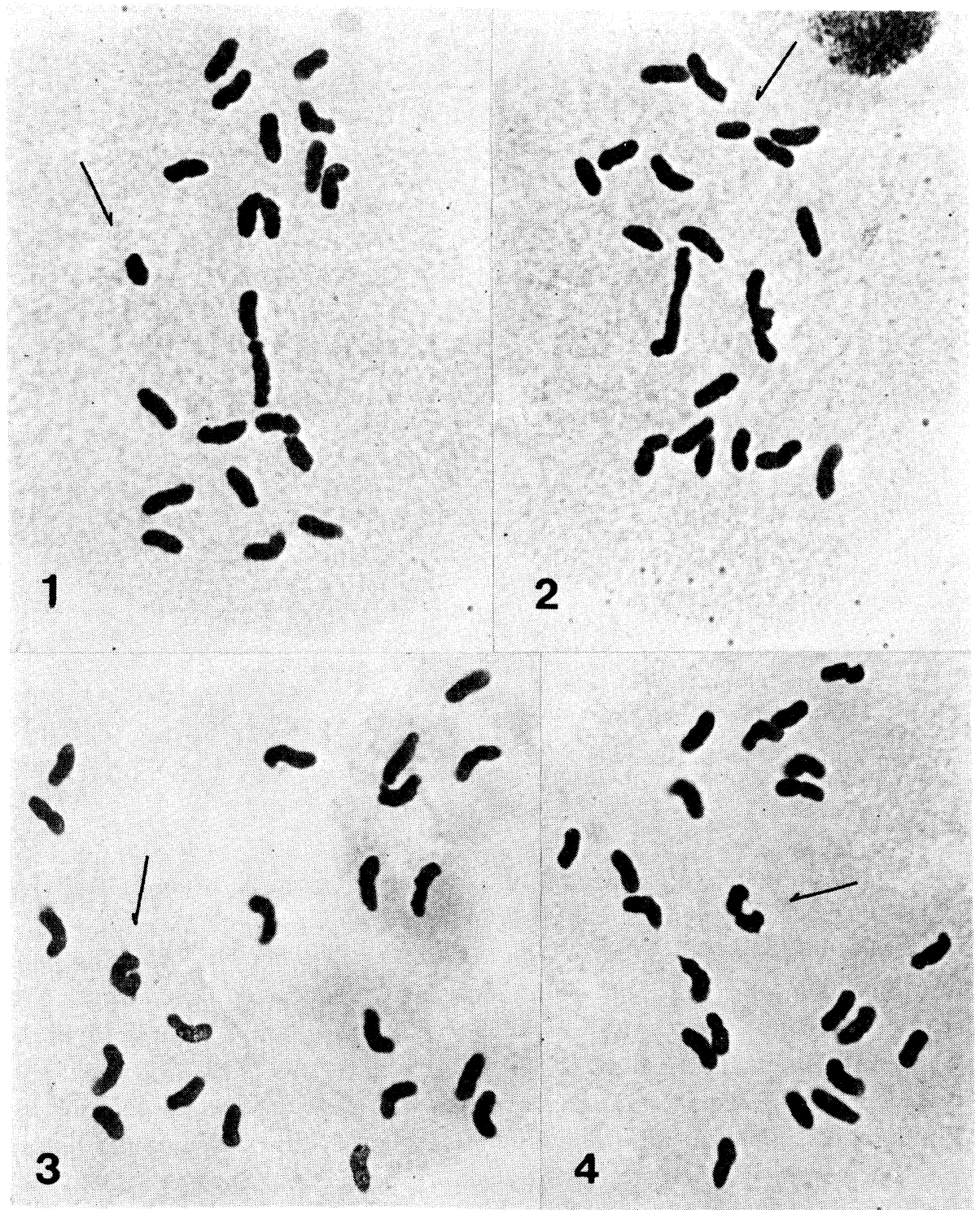

Figs. 1-4. Chromosome pairing at $\mathrm{MI}$ in Ae. speltoides $\times$ tetraploid wheat hybrids with B-chromosomes. $(\times 1250)$

Figs. 1 , 2. $\mathrm{S} \times \mathrm{AB}(7717 \times 110) ; 19_{\mathrm{I}}+1_{\mathrm{II}}+1 \mathrm{~B}(1), 17_{\mathrm{I}}+2_{\mathrm{II}}+1 \mathrm{~B}(2)$. Figs. $3,4 . \mathrm{S} \times \mathrm{AG}$ $(7717 \times 8601) ; 21_{I}+2 B$ 's $(3), 19_{I}+1_{I I}+2 B$ 's $(4)$. B-chromosomes are arrowed.

B's (7717), we could not find genotypes which led to such a low level of pairing showing 18 or more univalents per cell as was observed in the hybrids with 2 or 3 B's (Table 1). Therefore, the pairing observed may be non-homologous or homoeologous, as pointed out by Riley et al. (1961). The amount of homoeologous pairing observed seems to depend on the speltoides genotypes used. The strain 7712, for example, showed a high level of pairing in hybrids, while 7716 showed a low level of pairing 


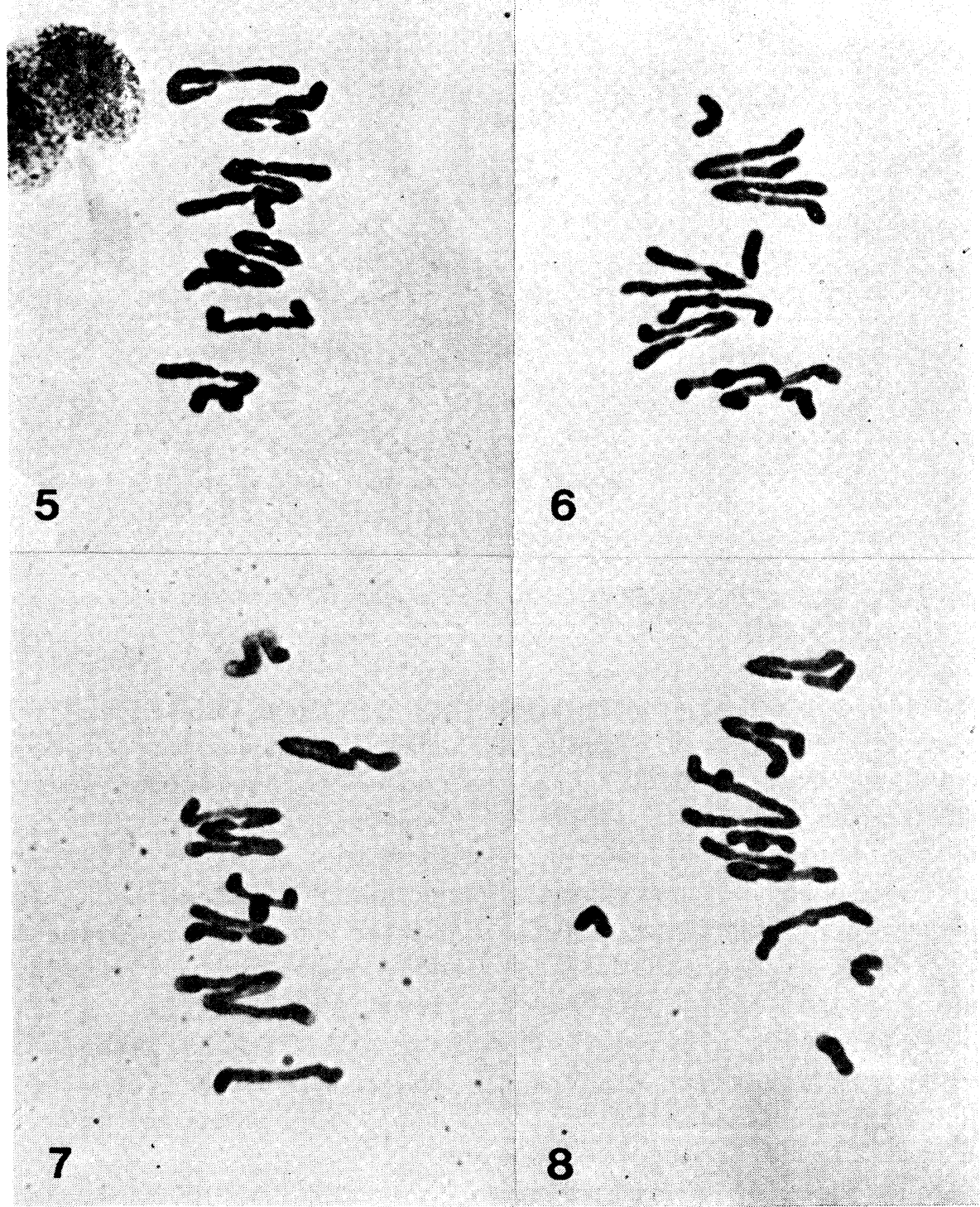

Figs. 5-8. Chromosome pairing at $\mathrm{MI}$ in Ae. speltoides $\times$ tetraploid wheat hybrids without B-chromosomes. $(\times 1250)$

Figs. 5 , 6. $\mathrm{S} \times \mathrm{AB}(7712 \times 110) ; 1_{\mathrm{I}}+2_{\mathrm{II}}+4_{\mathrm{III}}+1_{\mathrm{IV}}$ (5), $2_{\mathrm{I}}+2_{\mathrm{II}}+5_{\mathrm{III}} \quad$ (6). Figs. 7,8 . $\mathrm{S} \times$ AG $(7712 \times 8601) ; 2_{\mathrm{I}}+2_{\mathrm{II}}+1_{\mathrm{IV}}+1_{\mathrm{V}}(7), 3_{\mathrm{I}}+2_{\mathrm{II}}+2_{\mathrm{III}}+2_{\mathrm{IV}}(8)$.

(Table 2). No apparent difference due to the genomic constitution of the wheat parent was observed. Both the $\mathrm{AB}$ and $\mathrm{AG}$ genomes showed essentially the same amount of pairing in hybrids with Ae. speltoides (Table 2). But the SAG-hybrids showed a slightly higher frequency of multivalents from quadrivalents to septivalents, than the SAB-hybrids. The SAG-hybrids also showed different distribution (or terminalization) of chiasmata from the SAB-hybrids. The SAG-hybrids were observed to have more 

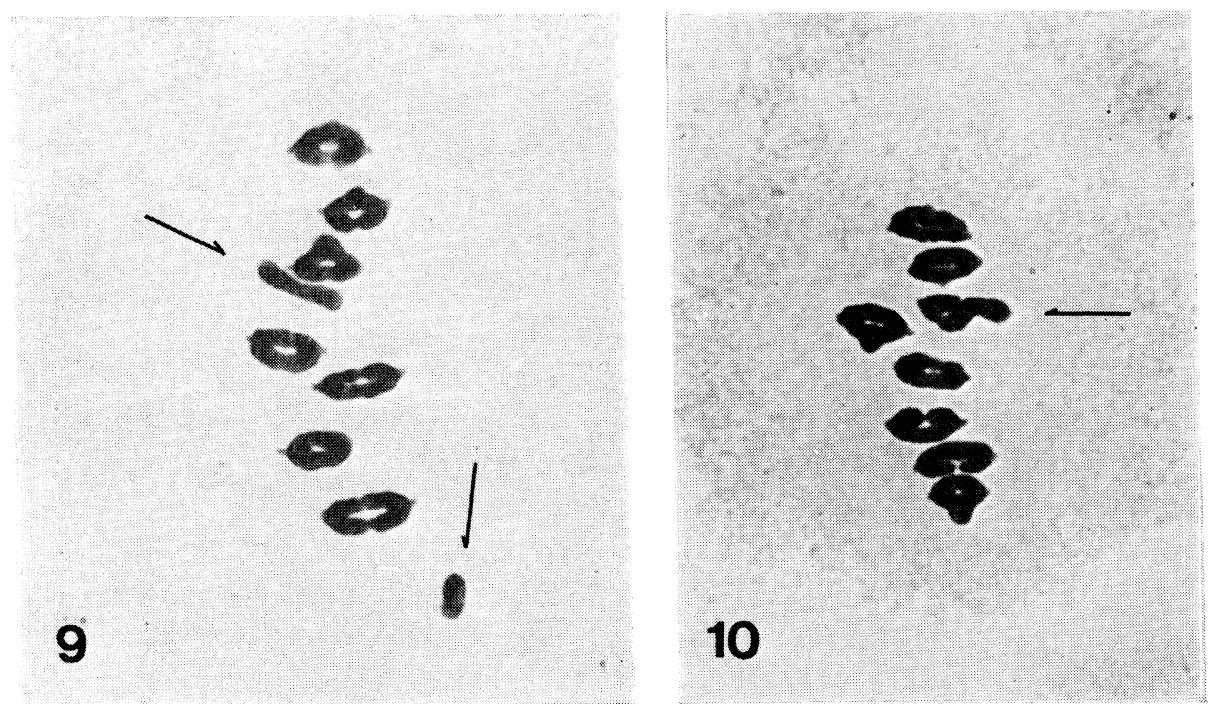

Figs. 9-10. Chromosome pairing at MI in Ae. speltoides (7717) with 3 B-chromosomes (arrowed). $\quad(\times 1250)$

interstitial-chiasmata than the SAB-hybrids (Table 2). These observations may show to some extent a difference of chromosomal homology or homoeology among the S, $\mathrm{A}, \mathrm{B}$, and $\mathrm{G}$ genomes, or the $\mathrm{AG}$ genomes may contain a weaker suppressor (s) of pairing than the $\mathrm{AB}$ genomes.

It is apparent from these data that B-chromosomes of Ae. speltoides induce a striking decrease in homoeologous pairing. Dosage effect of the B's seems to exist (Table 1). Genotypes of Ae. speltoides also have some effect on homoeologous pairing. Shands and Kimber (1973) reported a "low-pairing" genotype of Ae. speltoides, which led to a mean pairing of $20.20_{\mathrm{I}}+0.40_{\mathrm{II}}$ in a hybrid of $T$. durum $(\mathrm{AABB}) \times$ Ae. speltoides and $7.20_{\mathrm{I}}$ $+4.87_{\mathrm{II}}+1.55_{\mathrm{III}}+0.17_{\mathrm{IV}}$ in a hybrid of $T$. timopheevi (AAGG) $\times$ Ae. speltoides. They thought that these results showed a lack of homology between the $\mathrm{B}$ and $\mathrm{S}$ genomes and a high degree of homology between the $G$ and $S$. The former conclusion agrees with the present results but the latter does not.

Concerning the effect of B-chromosomes, Dover and Riley (1972b) observed that the presence of B's resulted in a marked reduction in the amount of homoeologous pairing in $5 \mathrm{~B}$ deficient hybrids of $T$. aestivum $\times$ Ae. speltoides. But when $5 \mathrm{~B}$ was present, the presence of B's did not always result in a striking decrease in homoeologous pairing. By the analogy from the results obtained by Dover and Riley (1972a) in hybrids without B's of $T$. aestivum $\times$ Ae. mutica, Vardi and Dover (1972) believed that the B's did not introduce factors altering the level of pairing other than that due to the genotypes of Ae. speltoides. It is very difficult, however, to explain the striking reduction of pairing in the present experiment by assuming such genotypes. The B-chromosomes must have a similar effect to the pairing suppressor $P h$ on $5 \mathrm{~B}$ which inhibits homoeologous pairing but permits homologous pairing. There is a similar pairing suppressor on 3D (Mello-Sampayo 1971). These pairing suppressors may obscure the effect of B's 
in $T$. aestivum (AABBDD) $\times A e$. speltoides hybrids. In fact, Dover and Riley (1972b) recognized the effect of B's in 5B deficient hybrids of $T$. aestivum $\times A e$. speltoides. The effect was also recognized in Ae. speltoides $\times$ tetraploid wheat hybrids which, of course, lack 3D, in the present study. Furthermore, Riley et al. (1973) recognized the effect in Ae. speltoides $\times T$. boeoticum (AA) hybrids which, naturally, lack both 5B and 3D. These pairing suppressors and the activity of the B-chromosomes may counteract the Ae. speltoides genotype inducing homoeologous pairing.

\section{LITERATURE CITED}

Bowman, J. G., and H. Thomas, 1973 B chromosomes and chromosome pairing in Lolium perenne $\times$ Festuca arundinacea hybrid. Nature New Biol. 245: 80-81.

Dover, G. A., and R. Riley, 1972a Variation at two loci affecting homoeologous meiotic chromosome pairing in Triticum aestivum $\times$ Aegilops mutica hybrids. Nature New Biol. 235: 61-62.

Dover, G. A., and R. Riley, 1972b Prevention of pairing of homoeologous meiotic chromosomes of wheat by an activity of supernumerary chromosomes of Aegilops. Nature 240: 159-161.

Dvořák, J., 1972 Genetic variability in Aegilops speltoides affecting homoeologous pairing in wheat. Can. J. Genet. Cytol. 14: 371-380.

Evans, G. M., and A. J. Macefield, 1972 Suppression of homoeologous pairing by B chromosomes in a Lolium species hybrid. Nature New Biol. 236: 110-111.

Kimber, G., and R. S. Athwal, 1972 A reassessment of the course of evolution of wheat. Proc. Natl. Acad. Sci. USA 69: 912-915.

Larsen, J., and G. Kimber, 1973 The effect of the genotype of Triticum speltoides on the pairing of homologous chromosomes. Can. J. Genet. Cytol. 15: 233-236.

Lilienfeld, F., and H. Kihara, 1934 Genomanalyse bei Triticum und Aegilops. V. Triticum Timopheevi Zhuk. Cytologia 6: 87-122.

Mello-Sampayo, T., 1971 Genetic regulation of meiotic chromosome pairing by chromosome 3D of Triticum aestivum. Nature New Biol. 230: 22-23.

Mochizuki, A., 1964 Further studies on the effect of accessory chromosomes on chromosome pairing. Japan. J. Genetics 39: 356. (Abstr. in Japan.)

Rees, H., 1963 Deoxyribonucleic acid and the ancestry of wheat. Nature 198: 108-109.

Riley, R., V. Chapman, and T. E. Miller, 1973 The determination of meiotic chromosome pairing. Proc. 4th Int. Wheat Genet. Symp., Columbia, Missouri, USA pp. 731-738.

Riley, R., G. Kimber, and V. Chapman, 1961 Origin of genetic control of diploid-like behavior of polyploid wheat. J. Hered. 52: 22-25.

Riley, R., J. Unrau, and V. Chapman, 1958 Evidence on the origin of the B genome of wheat. J. Hered. 49: 91-98.

Sarkar, P., and G. L. Stebbins, 1956 Morphological evidence concerning the origin of the B genome in wheat. Amer. J. Bot. 43: 297-304.

Shands, H., and G. Kimber, 1973 Reallocation of the genomes of Triticum timopheevii Zhuk. Proc. 4th Int. Wheat Genet. Symp., Columbia, Missouri, USA pp. 101-108.

Simchen, G., Y. Zarchi, and J. Hillel, 1971 Supernumerary chromosomes in the second outbreeding species of the wheat group. Chromosoma 33: 63-69.

Tanaka, M., and H. Ishii, 1973 Cytogenetical evidence on the speciation of wild tetraploid wheats collected in Iraq, Turkey, and Iran. Proc. 4th Int. Wheat Genet. Symp., Columbia, Missouri, USA pp. 115-121.

Vardi, A., and G. A. Dover, 1972 The effect of B chromosomes on meiotic and pre-meiotic spindles and chromosome pairing in Triticum/Aegilops hybrids. Chromosoma 38: 367-385. 